\title{
Nonlinear Principal Component Analysis as a Tool for the Evaluation of Customer Satisfaction
}

\author{
P. A. Ferrari and G. Manzi \\ Department of Economics, Business and Statistics, Università degli Studi di Milano, Italy \\ (Received April 2007, accepted November 2007)
}

\begin{abstract}
In this paper we examine the problem of setting-up a suitable indicator for the assessment of customer satisfaction. The proposed indicator is based on the nonlinear principal component analysis technique. Its properties are examined, and further analysis concerning its application to real data, the treatment of missing values and comparisons with other competitors is presented. Finally, findings with regard to data from an opinion survey are presented and discussed.
\end{abstract}

Keywords: Optimal scaling, principal component analysis, questionnaire fitting, rasch analysis, satisfaction indicators, stability analysis.

\section{Introduction}

$\mathrm{T}$ he knowledge of customer preferences is essential for maintaining and/or improving market shares. In particular, the measurement of customer satisfaction has gained an increasing attention among economists. By now it is generally accepted that customer satisfaction can not be directly quantified but should be extracted from other observed variables which are connected with different aspects of satisfaction itself. In order to have knowledge of customer satisfaction, survey questionnaires (respondents are asked to declare their degree of satisfaction about different aspects of the service or product) are used. Hence, statistical analysis of data from these surveys is carried out and measures of each aspect of overall satisfaction are obtained, even though this is rather troublesome to handle for many different reasons.

First of all, customer satisfaction is measured indirectly by means of a set of related variables, whose relevance and/or weight are indeterminate. In addition, observed variables frequently have an ordinal measurement scale which should be suitably dealt with. Therefore, the level of contentment is generally dependent on both expectation and individual features of respondents. Furthermore, surveys concern questions like "Is $X$ your best supplier?" or "Would you recommend $X$ to other companies?". This leads to subjective variables that express what people think [9] or, better, what people say. A rather considerable dissemination of measurement errors can emerge from the subjective nature of variables and the cognitive dissonance can affect data outcome with bad consequences on results efficacy as it has been recently addressed by [2].

To assess the customer satisfaction, many different methods have been produced in statistical literature. Two main approaches can be distinguished. The first one uses statistical models to estimate the relationship between the latent variable and the manifest variable and involves, among others, the structural equation models [3] by applying Partial Least Squares [20] (PLS, presented, for example, in [19]), or LISREL (LInear Structured 
RELationship) [12]. The second approach does not assume any model, but uses descriptive analysis adopting dimensionality reduction methods.

This paper places itself within the second approach and primarily deals with the setting-up of synthetic indicators of the level of satisfaction, on the basis of ordinal responses. In order to reach this purpose, a Nonlinear Principal Component Analysis (NLPCA) approach $[10,16]$ is proposed.

The NLPCA is also focused on evaluating the reliability of the method for the analysis of specific survey questionnaires where respondents are asked to declare whether or not they are satisfied with regard to different aspects of a provided service and, at the same time, with regard to the overall service.

The paper is laid out as follows. In Section 2 we describe the structure of the survey and the data under investigation. The setting-up of a synthetic measure for the level of satisfaction and its main features are described in Section 3. Further analysis concerning the procedure is presented in section 4 with regard to applications to real data, treatment of missing data and comparisons with other popular competitors. In Section 5 the procedure is applied to real data in order to delineate its applicative potentiality. Finally, Section 6 is dedicated to conclusions.

\section{The Questionnaire and the Resulting Dataset Used in the Analysis}

The questionnaire and the dataset used in our analysis are available on the website http://www.economia.unimi.it/projects/CSProject/. Being particularly interested in testing the truthfulness of respondents and in detecting latent variables forming the customer satisfaction, we choose this questionnaire because of its specific structure. Its aim is to evaluate a customer (company) satisfaction on a service provided by a company ABC, with reference to the following 10 different aspects: "Equipment", "Sales Support", "Technical Support", "Training", "Supplies and Media", "Pre Press/Workflow and Post Press Solutions", "Customer Portal (My ABC)", "Administrative Support", "Terms, Conditions and Pricing" and "Site Planning and Installation". For each aspect $Y_{j}, j=1, \ldots, 10$, a set of questions (items) $y_{j 1}, \ldots, y_{j T}, \max \{T\}=7$, concerning the level of satisfaction on different features of the aspect and a corresponding set of importance level or "weight questions" $w_{j 1}, \ldots, w_{j T}$ associated with the $y_{j t}-\mathrm{s}$ are listed, together with one (or two) final question(s) $Y_{j_{i}}^{*}$, concerning the overall satisfaction with aspect $Y_{j}$. Some sparse information questions are also provided. Possible answers to questions $y_{j t}$ are ranked from 1 to 5 in an increasing level of satisfaction, whereas the possible weight answers $w_{j t}$ are ranked from 1 to 3 (low $=1$, medium $=2$ and high=3), with a fourth possibility for not relevant or not applicable (N/A) statements. An example of this type of questions is the Administrative Support questionnaire section reported in Table 1 below.

An initial section $X_{I n i}$, dedicated to the "Overall satisfaction" with regard to the ABC Company and a final section $X_{F i n}$ dedicated to compare ABC with other companies are also included in the questionnaire. On overall, the questionnaire is composed by 81 questions and should be completed by a person individuated by her/his position into the company (1=Owner, 2=Management, 3=Technical Management, 4=Technical Staff, 5=Operator, $6=$ Administrator, $7=$ Other, please specify). Other information concerning location, age of the equipment and the level of profitability is also gathered.

The resulting dataset is also suitable for missing data analysis because only 1 out of 266 respondents answered to all the questions without not relevant or not available answers. Because of the sparseness of questions we excluded from our analysis the following aspects: 
"Pre Press/Workflow and Post Press Solutions", "Customer Portal (My ABC)" and "Site, Planning and Installation".

Table 1. Administrative support section of the questionnaire.

\begin{tabular}{|c|c|c|c|}
\hline $\begin{array}{l}\text { No. of } \\
\text { question }\end{array}$ & Question - Item & Evaluation & Importance Level \\
\hline 50 & Invoices are provided on time. & $\begin{array}{l}\text { Strongly disagree }=1, \\
2,3,4,5=\text { Strongly agree }\end{array}$ & Low $=1,2,3=$ High, N/A \\
\hline 51 & $\begin{array}{l}\text { Invoices are correct when first } \\
\text { received. }\end{array}$ & $\begin{array}{l}\text { Strongly disagree }=1, \\
2,3,4,5=\text { Strongly agree }\end{array}$ & Low $=1,2,3=$ High, N/A \\
\hline 52 & $\begin{array}{l}\text { Invoices are clear and easy to } \\
\text { understand. }\end{array}$ & $\begin{array}{l}\text { Strongly disagree }=1, \\
2,3,4,5=\text { Strongly agree }\end{array}$ & Low $=1,2,3=$ High, N/A \\
\hline 53 & Credits are issued promptly. & $\begin{array}{l}\text { Strongly disagree }=1, \\
2,3,4,5=\text { Strongly agree }\end{array}$ & Low $=1,2,3=$ High, N/A \\
\hline 54 & Complaints are handled promptly. & $\begin{array}{l}\text { Strongly disagree }=1, \\
2,3,4,5=\text { Strongly agree }\end{array}$ & Low $=1,2,3=$ High, N/A \\
\hline 55 & $\begin{array}{l}\text { Administrative personnel are } \\
\text { friendly and courteous. }\end{array}$ & $\begin{array}{l}\text { Strongly disagree }=1, \\
2,3,4,5=\text { Strongly agree }\end{array}$ & Low $=1,2,3=$ High, N/A \\
\hline 56 & $\begin{array}{l}\text { When you have an administrative } \\
\text { problem, you know who to } \\
\text { contact. }\end{array}$ & $\begin{array}{l}\text { Strongly disagree }=1, \\
2,3,4,5=\text { Strongly agree }\end{array}$ & Low $=1,2,3=$ High, N/A \\
\hline 57 & $\begin{array}{l}\text { Overall satisfaction level with } \\
\text { administrative support. }\end{array}$ & $\begin{array}{l}\text { Strongly disagree }=1, \\
2,3,4,5=\text { Strongly agree }\end{array}$ & Low $=1,2,3=$ High, N/A \\
\hline
\end{tabular}

\section{A Proposal for the Analysis of Customer Satisfaction}

\subsection{The Setting-Up of a One-Dimension Indicator}

As we have already recalled, the level of satisfaction is a clear example of a phenomenon which can not be directly measured but can be evaluated by means of several variables which describe different aspects and, for every aspect, different levels of the same onedimensional phenomenon. In addition, these variables are categorical ones and usually are measured at an ordinal level scale (Likert scale) [13]. Sometimes the ordinal categories are coded in points, so that this adoption might result in a less subjective scale, but does not solve the problem of ordinal data. In fact the numerical labels indicate the rating of categories but not their values. Consequently, the numerical distance between subsequent labels does not reflect the real distance in an evaluation scale. It is then evident that data have to be analyzed with caution if we require that findings are not to be affected by unrealistic assumptions.

In order to achieve a suitable solution to these problems we assume the basic hypothesis that the measure of customer satisfaction can be obtained by reducing the dimensionality of multiple items indicating the different aspects or services. In other words, we assume that 
observations on the set of variables for each respondent can be mapped onto a low-dimensional variable (sometimes a one-dimensional variable that expresses her/his level of satisfaction).

To reach this goal we adopt NLPCA. This analysis belongs to the so-called "Gifi system" [10] and was presented by the Data Theory Group of the University of Leiden in 1990 and developed in the following years.

NLPCA for data on customer satisfaction seems particularly suitable because it allows synthesizing observed variables in a reduced space, preserving measurement levels of qualitative ordinal data without assuming an a priori difference between subsequent categories. The latent dimension is derived as a linear combination of the observed variables after an optimal quantification of their ordinal categories and of the weights to be assigned to each variable in the construction of the linear combination.

By NLPCA, each column of the data matrix (each ordinal variable) is monotonically transformed in such a way that a reduced number $p$ of new continuous variables (components) optimally fits the transformed data. Obviously $p=1$ if one needs only one single indicator (in our case, the level of satisfaction) but the use of more than one component can turn out to be useful in some situations as we will show in our applications.

In the one dimensional case, when $m$ ordinal variables are observed without missing observations on $n$ objects (here variables are items of the questionnaires and objects are respondents), NLPCA can be formalized as follows.

Let $\mathbf{c}_{j}$ the $k_{j}$-dimensional vector containing the ordinal categories of the $j$ th variable, $j=1,2, \ldots, m, \mathbf{H}$ the $n \times m$ matrix containing the observations of the $m$ variables on the $n$ objects, $\mathbf{h}_{j}$ the $j$ th column of the matrix $\mathbf{H}, \mathbf{G}_{j}$ the $n \times k_{j}$ indicator matrix such that $\mathbf{G}_{j} \mathbf{c}_{j}=\mathbf{h}_{j}$. The target of NLPCA is to find the vector $\mathbf{x}_{[n \times 1]}$ of object scores (here interpreted as respondents' satisfaction measures) that minimizes the following loss function:

$$
\sigma^{2}\left(\mathbf{x} ; \mathbf{q}_{1}, \ldots, \mathbf{q}_{m} ; \beta_{1}, \ldots, \beta_{m}\right)=\frac{1}{m} \sum_{j=1}^{m}\left(\mathbf{x}-\mathbf{G}_{j} \mathbf{q}_{j} \beta_{j}\right)^{T}\left(\mathbf{x}-\mathbf{G}_{j} \mathbf{q}_{j} \beta_{j}\right)
$$

where $\mathbf{q}_{j}(j=1,2, \ldots, m)$ is the $k_{j}$ vector that contains optimal category quantifications for variable $j$ and $\beta_{j}$ is a scalar of component loading for variable $j$.

In order to avoid trivial solutions, identification constraints are required. Usually, object scores and transformed variables are standardized, so the following conditions are imposed:

$$
\begin{aligned}
& \mathbf{x}^{T} \mathbf{x}=n \\
& \mathbf{u}_{n}^{T} \mathbf{x}=0,
\end{aligned}
$$

with $\mathbf{u}_{n}^{T}$ vector of ones of order $n$; but other normalization methods can be adopted.

A further condition is $\mathbf{q}_{j} \in C_{j}$, where $C_{j}$ is the convex cone of vectors with non-decreasing elements.

The optimal solution is derived by means of an iterative algorithm called Alternating Least Squares (ALS), conveniently adapted to this case to assure also on the order of quantifications $[10,16]$. 
The optimal scaling level here discussed is the Ordinal one, but other solutions can be adopted, in particular the Spline Ordinal can result suitable for these data ([17], see section $5)$.

The one dimensional solution obtained provides the following object scores:

$$
\mathbf{x}=\frac{1}{m} \sum_{j} \mathbf{G}_{\mathbf{j}} \mathbf{q}_{j} \beta_{j}=\frac{1}{m} \sum_{j} \mathbf{t}_{j} \beta_{j}
$$

where $\mathbf{t}_{j}$ is the $n \times 1$ vector of the transformed variable $j$.

Therefore, given the standardisation conditions, it also follows that, if no missing data affect the data-set, component loadings $\beta_{j}$ are correlations between object scores and optimally quantified variables.

\subsection{The One Dimensional Solution of NLPCA as an Indicator of Customer Satisfaction}

Scores by NLPCA may be used as measures of customer satisfaction. In fact, by formula (3), a quantitative value obtained as weighted mean of transformed variables with loadings $\beta_{j}$ as weights is assigned to each respondent. In such a way, the value obtained for each respondent measures her/his level of satisfaction. Nevertheless, before using the one-dimensional solution as a feasible indicator of satisfaction, it is necessary to evaluate its validity. With this in mind, we need to ascertain the following requirements:

(i). the first eigenvalue of the NLPCA solution is effectively much larger than the others and the solution itself fits well the data;

(ii). all the weights should have the same sign;

(iii). the solution is stable.

As in the PCA, the first eigenvalue of the NLPCA constitutes a measure of goodness of fit of the procedure. In fact, the goodness of an indicator depends on the minimization of the sum of the squared distances between the obtained scores and the data. In order to evaluate the goodness of the procedure it is thus possible to use the first eigenvalue $\lambda_{1}$ of the correlation matrix of transformed variables or, better, a percentage ratio between $\lambda_{1}$ and $m$ (the number of variables in the dataset), known as the percentage of total variance accounted for by the first dimension: the larger is the ratio, the better is the synthesis.

Alternatively, Cronbach's $\alpha$ [4] can be determined. This coefficient, introduced as a tool for assessing the reliability of scales, is strictly connected with $\lambda_{1}$ [11] by the following:

$$
\alpha=\frac{m\left(\lambda_{1}-1\right)}{(m-1) \lambda_{1}} .
$$

With regard to point (ii) above, since the vector of object scores $\mathbf{x}$ is built as a simple linear combination of quantified categories, each $\mathbf{x}_{i}$ has to fulfil the mathematical conditions for a linear index to be valid, in the sense that the higher is the rank of observed variables, the higher is the value of the satisfaction indicator. This requires that the weights of combinations have the same sign, specifically the positive one, for each variable.

Finally, it is also important to evaluate the stability of the outputs produced (i.e. eigenvalues, component loadings, category quantifications and average scores). This is a topic still under tight scrutiny of the researchers and no definitive solutions have been presented so far. 
One of these solutions is the evaluation of stability by means of resampling methods, which in general avoid troublesome theoretical proofs. In particular, among the various resampling methods, the bootstrap method is an empirical and easy way to suitably test whether or not the indicators resulting from NLPCA are more or less stable.

The bootstrap technique was first proposed in order to evaluate bias and variance of an estimator of a parameter of interest [5,6]. The basic idea lies on the fact that the empirical distribution function $\hat{F}$ is a good estimate of the underlying and unknown population with distribution function $F$. The evaluation of the estimator stability is based on the construction of a bootstrap population by repeatedly resampling from an original i.i.d. sample. The computation of the entire real bootstrap population can be theoretically done, but computational problems arise quite soon: so that instead of drawing all the possible resamples, it is generally accepted to draw a lower number $B$ of resamples and then evaluate the stability on the basis of this set of resamples.

In the NLPCA context, the bootstrap method can be used to check the stability of all the outputs. For example, to verify the component loadings stability, an algorithm which consists of bootstrapping samples with replacement from the data set can be adopted, in this way producing also bootstrap confidence intervals so as usually defined by the classical bootstrap method literature.

\section{Further Considerations on the Procedure}

Before passing to the applications we discuss in this section further features of NLPCA.

\subsection{Further Analysis}

Once the optimal quantifications and weights are determined and validated, the $n \times m$ matrix $\mathbf{T}=\left[\mathbf{t}_{1}|\ldots| \mathbf{t}_{m}\right]=\left[\mathbf{G}_{1} \mathbf{q}_{1}|\ldots| \mathbf{G}_{m} \mathbf{q}_{m}\right]$ of transformed data may be considered for further statistical analysis: in such a way it is possible to analyse the level of satisfaction according to specific factors which could be in a particular relationship with it.

For example, if one is interested to compare the level of satisfaction in different countries, the procedure to compute the indicator at the country level is the following. Let $\zeta$ be the index of the country and $n_{\zeta}$ the number of respondents within country $\zeta\left(\sum_{\zeta} n_{\zeta}=n=\right.$ total sample size $)$. It is possible to compute the conditional mean of scores $\bar{x}_{\zeta}$ for country $\zeta$, which is given by:

$$
\bar{x}_{\zeta}=\frac{1}{n_{\zeta}} \mathbf{u}_{\zeta}^{T} \mathbf{x}
$$

where $\mathbf{u}_{\zeta}^{T}$ is an indicator vector of order $n$ with a block of $n_{\zeta}$ ones in the position corresponding to country $\zeta$ and zeros elsewhere and $\mathbf{x}$ is the $n \times 1$ vector of scores. mean is:

Values of $\bar{x}_{\zeta}$ can be directly used for comparison. In fact, since the grand population

$$
\bar{x}=\frac{1}{n} \sum_{\zeta} n_{\zeta} \bar{x}_{\zeta}=0
$$

then $\bar{x}_{\zeta} \geq 0(<)$ denotes equal or greater (lower) average satisfaction of the country with respect to the average and it measures the country effect. 
Furthermore we can use these quantifications to analyze the correlation between different items or between different aspects of the service provided and so forth.

In addition we can not stop the analysis at the first component but continue in extracting dimensions $p>1$. In this case, formula (1) assumes the more general expression:

$$
\sigma^{2}\left(\mathbf{X} ; \mathbf{q}_{1}, \ldots, \mathbf{q}_{m} ; \beta_{1}, \ldots, \beta_{m}\right)=\frac{1}{m} \sum_{j=1}^{m}\left(\mathbf{X}-\mathbf{G}_{j} \mathbf{q}_{j} \beta_{j}\right)^{T}\left(\mathbf{X}-\mathbf{G}_{j} \mathbf{q}_{j} \beta_{j}\right)
$$

being $\mathbf{X}$ the $n \times p$ matrix containing the object scores for the $p$ latent variables and $\beta_{j}$ the $p$-dimensional vector of component loadings. Formulas in (2) become

$$
\begin{aligned}
\mathbf{X}^{T} \mathbf{X} & =n \mathbf{I} \\
\mathbf{u}_{n}^{T} \mathbf{X} & =\mathbf{0},
\end{aligned}
$$

whereas the percentage of total variance accounted for by the first $p$ dimensions is given by $\left(\sum_{j=1}^{p} \lambda_{j} / m\right) \times 100$. It is also worthy noting that, given a $p$ dimensional solution optimal quantifications are the same in every dimension but, contrary to PCA, solutions for $1,2, \ldots$, $p$-dimensions are not nested.

In section 5 some examples are given.

\subsection{Missing Data}

When statistical data are collected with survey questionnaires, many missing data are often recorded. The problem of missing data is well known in statistical literature and many methods are available for their treatment [14].

With regard to NLPCA, the following three standard procedures are presented in [10]: (a) passive; (b) simple category; (c) multiple categories.

The first one does not take missing observations into consideration for computation, whereas the last two options require strong assumptions about the pattern of missing data. More specifically, in case (a), the indicator matrix is left incomplete, the generic row of $\mathbf{G}_{j}$ is zero if the corresponding unit has one missing observation for variable $j$.

Formula (1) is generalized in the following way:

$$
\sigma^{2}\left(\mathbf{x} ; \mathbf{q}_{1}, \ldots, \mathbf{q}_{m} ; \beta_{1}, \ldots, \beta_{m}\right)=\frac{1}{m} \sum_{j=1}^{m} \operatorname{tr}\left(\mathbf{x}-\mathbf{G}_{\mathrm{j}} \mathbf{q}_{j} \beta_{j}\right)^{T} \mathbf{M}_{j}\left(\mathbf{x}-\mathbf{G}_{\mathrm{j}} \mathbf{q}_{j} \beta_{j}\right)
$$

where $\mathbf{M}_{j}$ is a binary diagonal matrix of order $n \times n$ which detects missing observations for variable $j, \mathbf{M}_{j}(i, i)$ is equal to 1 if the $i$-th observation is present for the $j$-th variable, and 0 otherwise. In this case, normalization restrictions and object scores become the following [16]:

$$
\begin{gathered}
\mathbf{x}^{T} \mathbf{M} \mathbf{x}=m n \mathbf{I}, \quad \mathbf{u}^{T} \mathbf{M} \mathbf{x}=0 \\
\text { with } \mathbf{M}=\sum_{j=1}^{m} \mathbf{M}_{j}, \text { and } \\
\mathbf{x}=\mathbf{M}^{-1} \sum_{j=1}^{m} \mathbf{G}_{j} \mathbf{q}_{j} \beta_{j} .
\end{gathered}
$$


In the other two options, the indicator matrix is completed for variable $j$, with an additional column with one entry for the units with missing observations (case (b)), or with several columns with one entry for each unit with missing observations (case (c)). In the last two options, missing data are treated as categories themselves [7, 10].

\subsection{NLPCA vs. Main Competitors}

In this section we briefly discuss two other methods that belong to different approaches and give measures of the latent trait underlying a multiple-item scale when the target is to reach the same objective in the context we are treating. They are: the PCA and the Rasch Model (RM). The first one is connected to an algorithmic procedure according to which no generating data process is assumed but the best representation of the data is searched; the second one assumes a model entirely known except for the values of parameters which have to be estimated.

The PCA consists, as the NLPCA, in a reduction process that sets up the indicator as a linear combination of manifested variables. To carry out the analysis it is necessary that the observed variables are numerical ones. In the case of ordinal variables, the categories of the variables are requested to be coded in points before the analysis. This, contrary to NLPCA, implies the adoption of an a priori difference between subsequent categories. Furthermore, PCA assumes a linear relation among the observed variables which could not be realistic. Hence this very popular method appears not particularly suitable when these conditions are not accomplished. In section 5 we will present results which show the unsuitability of PCA.

The Rasch Model was introduced by Rasch as a psychometric tool to measure both the item difficulty and the subject ability along a shared continuum, on the basis of the answer to the items of a questionnaire [18]. Originally proposed for dichotomous responses, it was later extended to polytomous ordinal responses according to the following two versions: the Rating Scale Model (RSM) [1] and the Partial Credit Model (PCM) [15, 21]. The RSM assumes that each item has the same rating structure, whilst for the PCM this structure can vary.

The Rasch Model, in the more suitable version, can be used alternatively to NLPCA to reach similar objectives (see, for example, [8]). Although based on different premises, both methods give scores for every respondent, which can be assumed as measures of her/his satisfaction, as well as distances between categories of items. Nevertheless NLPCA seems more suitable in some contexts for two reasons. First, since NLPCA gives the level of satisfaction as a linear combination of quantified questionnaire answers, then it is immediate to compute the score of any customer-to-be. Secondly, while for the Rasch Model unidimensionality is a fundamental assumption and, hence, only one dimension can be considered, for the NLPCA it is possible to contemplate more than one dimension, enriching the analysis on satisfaction if needed.

\section{Applications}

In the previous sections we discussed the possibility of adopting the NLPCA analysis to assess the customer satisfaction. The NLPCA procedure, its main characteristics and the ambit of application were also discussed. Now we show how it can be applied to the ABC survey data and what are the main advantages deriving from its application. Many findings can be derived from this application so that we limit our account to delineate only some directions of analysis. 
For the sake of brevity we do not describe results in depth, because they derive directly from what we have pointed out in the above sections. We rather sketch them in figures or tables so that they get more evident. For the same reason we present only attained findings without missing data.

\subsection{Setting-Up of the Indicator}

The NLPCA has been applied to the data set and indicators of customer satisfaction are carried out for each of the seven aspects considered and for the overall questions. Table 2 shows the variance accounted for by the first component, the Cronbach's $\alpha$, the range of component loadings and the range of quantifications of single questions for all the seven aspects and for the seven overall questions considered together. For each aspect and for the overall questions, items of the data set with one or more missing answers were erased. The resulting size is illustrated in the second column of the table.

Table 2. First component variance, Cronbach's $\alpha$, number of items, loadings ranges.

\begin{tabular}{|l|r|r|r|r|c|}
\hline \multicolumn{1}{|c|}{ Aspect } & $\begin{array}{c}\text { No. of } \\
\text { cases }\end{array}$ & $\begin{array}{c}\text { \% of } \\
\text { variance }\end{array}$ & $\begin{array}{c}\text { No.of } \\
\text { items }\end{array}$ & $\begin{array}{c}\text { Cronbach's } \\
\alpha\end{array}$ & $\begin{array}{c}\text { Component } \\
\text { loadings range }\end{array}$ \\
\hline Equipment & 66 & 47.51 & 5 & 0.724 & $0.336-0.869$ \\
\hline Sales Support & 218 & 72.43 & 5 & 0.905 & $0.821-0.884$ \\
\hline Technical Support & 245 & 61.95 & 7 & 0.898 & $0.542-0.898$ \\
\hline Training & 108 & 73.53 & 5 & 0.910 & $0.822-0.884$ \\
\hline Supplies and Media & 42 & 62.30 & 6 & 0.879 & $0.473-0.914$ \\
\hline Administrative Support & 178 & 61.90 & 7 & 0.897 & $0.668-0.865$ \\
\hline Terms, Conditions and Pricing & 104 & 49.82 & 7 & 0.832 & $0.523-0.842$ \\
\hline Overall questions & 144 & 46.15 & 7 & 0.805 & $0.637-0.725$ \\
\hline
\end{tabular}

Table 2 shows that, for each aspect, the one-dimension component fits well the data having the component loadings always the same sign. Furthermore, their ranges strongly are different each other. This suggests that it is not suitable to assume the same weight in considering the different answers when constructing a structured customer satisfaction indicator. The resulting values of component loadings detect the presence of hotspots. So, for example, the very low value of 0.336 corresponding to item "Uptime is acceptable" of the Equipment aspect suggests the existence of some problems with this item.

In Figure 1 the quantifications resulted from the NLPCA analysis are compared with the possible answers (real categories) to the seven overall questions on the chosen aspects. It can be noted that the hypothesis of equal distance between categories does not hold, and that for several items some of the categories are not significantly different (see, for example, the cases of category 1 and category 2 of Equipment, Technical Support and Training aspects). This is confirmed considering the specific items for each aspect, as shown in Figure 2 (see the interesting cases of the Equipment aspect, the Technical Support and the Supplies and Media aspect, for which there is no linear relationship between actual categories and NLPCA quantifications). 

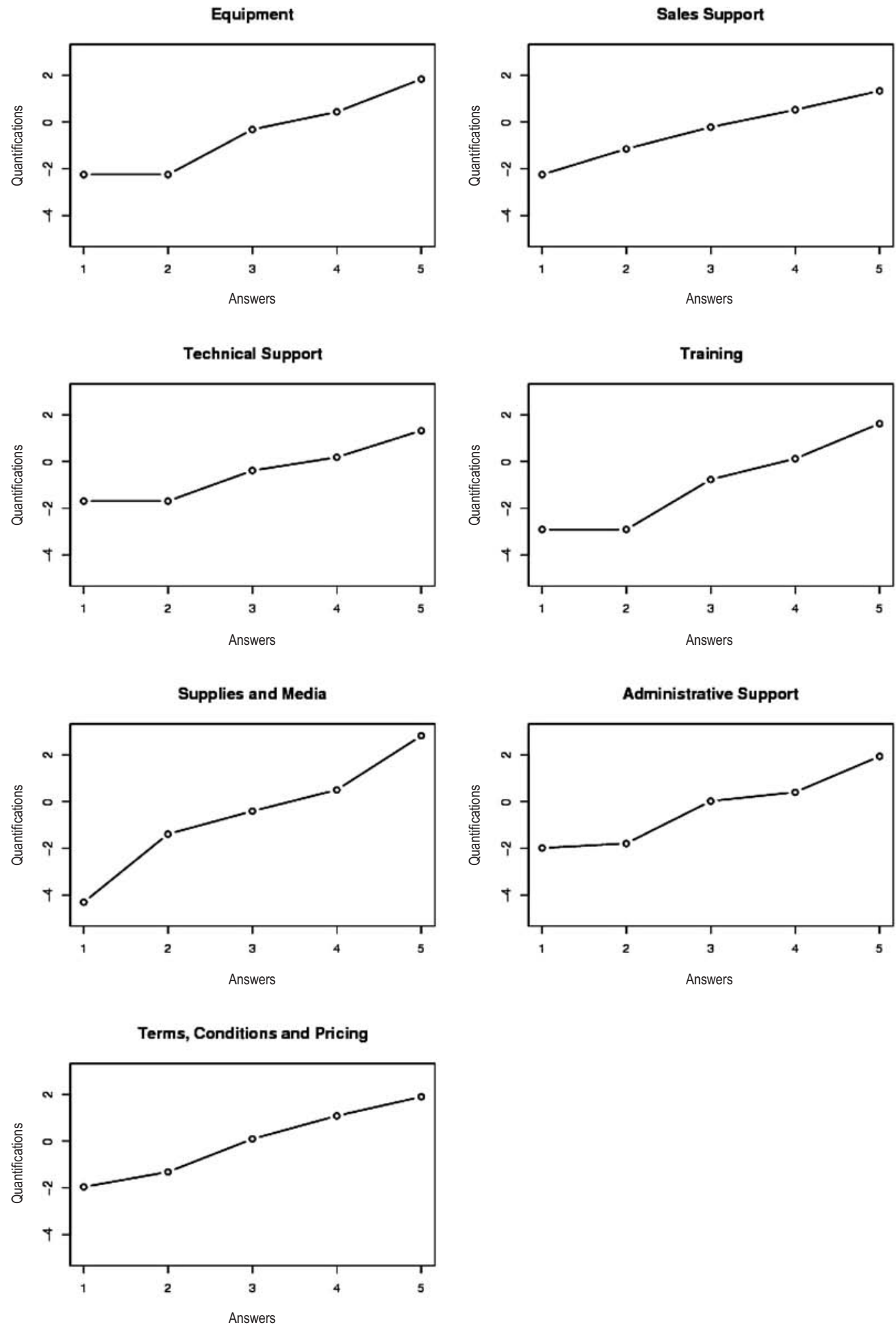

Figure 1. NLPCA quantifications for the overall questions vs. answers of respondents. 


\subsection{NLPCA vs. Main Competitors}

In Table 3 a comparison between NLPCA and PCA results in the case of Supplies and Media is sketched. The percentage of variance accounted is $42 \%$ in PCA and $67 \%$ in NLPCA, resulting in a considerable increase in model fitting in favour of NLPCA. This is due to the nonlinearity of the relationship as it can be noted by the comparison between PCA scale points and optimal scaled NLPCA quantifications, stressed in the same table. On the contrary, component loadings values are similar both in NLPCA and in PCA.

Table 3. PCA vs. NLPCA: quantifications and component loadings for aspect Supplies and Media.

\begin{tabular}{|c|c|c|c|c|c|c|c|}
\hline \multicolumn{8}{|c|}{ PCA categories vs. NLPCA quantifications } \\
\hline \multirow{2}{*}{ Question 32} & PCA & NA & 2 & 3 & 4 & \multicolumn{2}{|c|}{5} \\
\hline & NLPCA & NA & -2.67 & 0.19 & 0.56 & \multicolumn{2}{|c|}{0.88} \\
\hline \multirow{2}{*}{ Question 33} & PCA & 1 & 2 & 3 & 4 & \multicolumn{2}{|c|}{5} \\
\hline & NLPCA & -2.22 & -2.22 & 0.40 & 0.40 & \multicolumn{2}{|c|}{0.87} \\
\hline \multirow{2}{*}{ Question 34} & PCA & 1 & 2 & 3 & 4 & \multicolumn{2}{|c|}{5} \\
\hline & NLPCA & -2.61 & -2.61 & 0.41 & 0.41 & \multicolumn{2}{|c|}{1.86} \\
\hline \multirow{2}{*}{ Question 35} & PCA & 1 & 2 & 3 & 4 & \multicolumn{2}{|c|}{5} \\
\hline & NLPCA & -3.70 & -1.76 & 0.22 & 0.22 & \multicolumn{2}{|c|}{1.03} \\
\hline \multirow{2}{*}{ Question 36} & PCA & 1 & 2 & 3 & 4 & \multicolumn{2}{|c|}{5} \\
\hline & NLPCA & -4.35 & -1.89 & 0.38 & 0.38 & \multicolumn{2}{|c|}{0.38} \\
\hline \multirow{2}{*}{ Question 37} & $\mathrm{PCA}$ & 1 & 2 & 3 & 4 & \multicolumn{2}{|c|}{ NA } \\
\hline & NLPCA & -3.85 & -1.86 & 0.43 & 0.43 & & \\
\hline \multicolumn{8}{|c|}{ PCA vs. NLPCA component loadings } \\
\hline & & $\begin{array}{c}\text { Question } \\
32 \\
\end{array}$ & $\begin{array}{c}\text { Question } \\
33\end{array}$ & $\begin{array}{c}\text { Question } \\
34\end{array}$ & $\begin{array}{c}\text { Question } \\
35\end{array}$ & $\begin{array}{c}\text { Question } \\
36\end{array}$ & $\begin{array}{c}\text { Question } \\
37 \\
\end{array}$ \\
\hline & PCA & 0.846 & 0.739 & 0.397 & 0.539 & 0.717 & 0.839 \\
\hline & NLPCA & 0.914 & 0.855 & 0.473 & 0.687 & 0.805 & 0.909 \\
\hline
\end{tabular}

The main advantage of the NLPCA, in comparison with the Rasch model, is that in NLPCA one can extract more than one latent dimension, and, doing so, can describe different aspects of customer satisfaction. For example, with regard to the Administrative Support, aspect results are sketched in Figure 3. It can be observed that, independently from the overall level, the satisfaction can be affected in different ways as the sketch of the second component highlights. We can note that the loadings with positive sign, concerning the first three items, are connected with administrative issues, whereas the ones with negative sign are the last four which are connected with relation issues. This means that for one single respondent, the larger is the value of this component, the more relevant is the aspect in examination (see Table 4), and this is true independently from the chosen normalization method for object scores and variables (variable principal, object principal, symmetrical or independent normalization - see also Figure 3). 

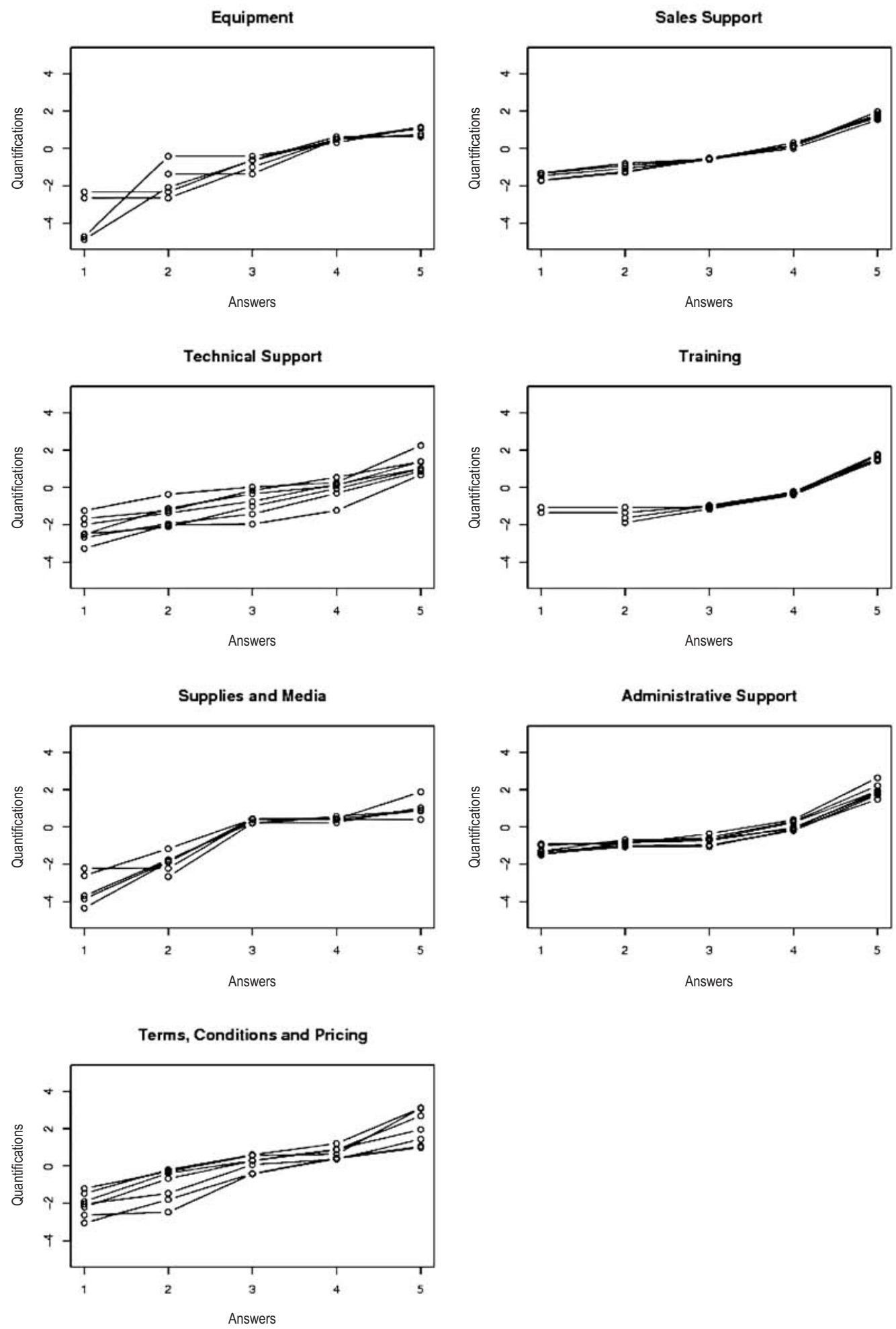

Figure 2. NLPCA quantifications for questions included in aspect sections vs. answers by respondents. 


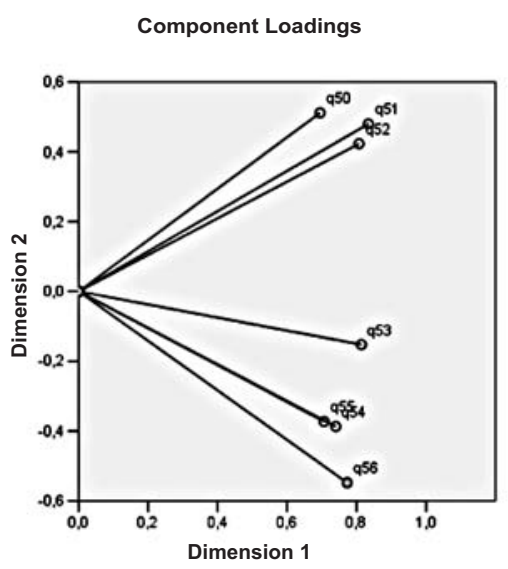

Variable Principal Normalization.

Component Loadings

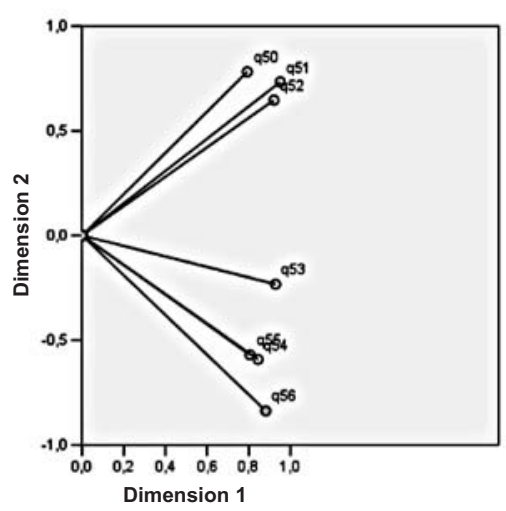

Symmetrical Normalization.

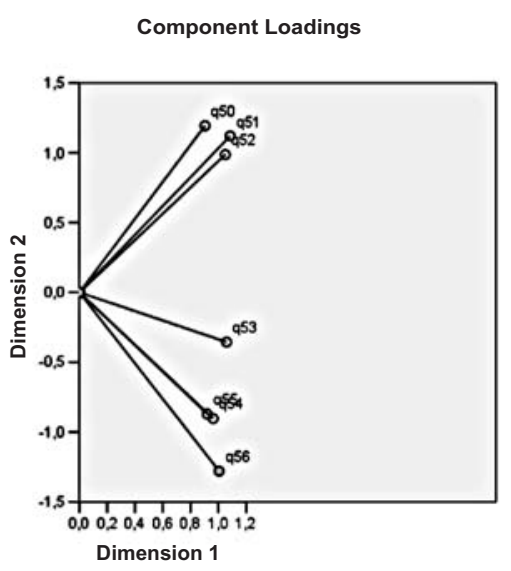

Object Principal Normalization.

Component Loadings

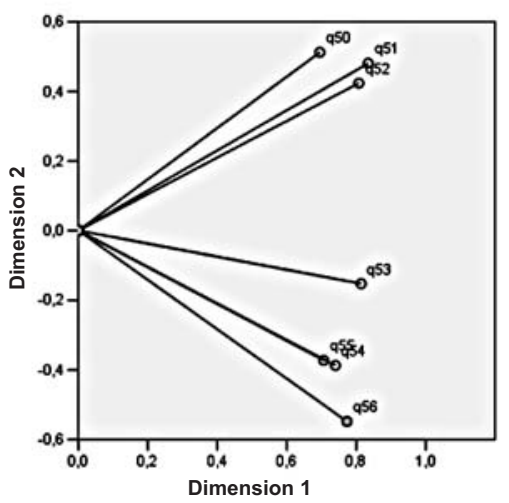

Independent Normalization.

Figure 3. Component loadings of the first and second dimension for the items of Administrative Support and different normalization method.

Table 4. Component loadings for the first and second dimension for the items of administrative support aspect.

\begin{tabular}{|c|c|c|}
\hline \multirow{2}{*}{} & \multicolumn{2}{|c|}{ Dimension } \\
\cline { 2 - 3 } & 1 & 2 \\
\hline Question no. 50 & 0.628 & 0.567 \\
\hline Question no. 51 & 0.826 & 0.299 \\
\hline Question no. 52 & 0.775 & 0.402 \\
\hline Question no. 53 & 0.871 & -0.066 \\
\hline Question no. 54 & 0.780 & -0.374 \\
\hline Question no. 55 & 0.725 & -0.335 \\
\hline Question no. 56 & 0.811 & -0.397 \\
\hline
\end{tabular}




\subsection{Further Analysis}

The overall level of satisfaction as well as the level of satisfaction on every aspect or single item can be analyzed with regard to every factor which could affect them. In Figure 4 the average level of the overall satisfaction for each country involved in the analysis is shown. The values have been obtained with (4) on the seven overall questions. No substantial differences among object scores were detected using different scales of quantifications. Two different scaling levels to quantify each variable were compared: the spline ordinal and the ordinal. In terms of percentage of variance accounted for and of Crombach's $\alpha$, the solutions derived from the two scaling levels are very similar: for example, in the case of the overall questions considered together, Cronbach's $\alpha$ is equal to 0.804 using the spline ordinal scaling level and to 0.809 using the ordinal scaling level.

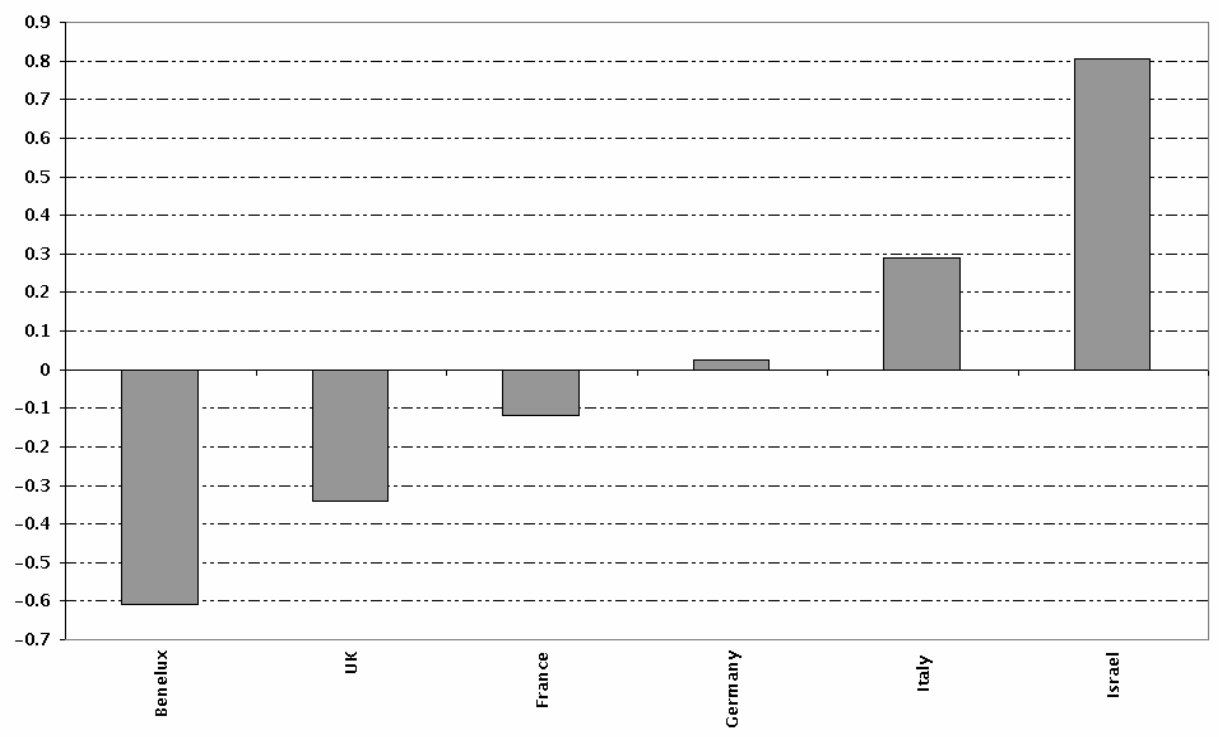

Figure 4. Averages of object scores by country. Overall questions on the seven aspects considered.

In addition, a bootstrap-percentile method could be used to check the variability of indicators. The bootstrap method is required because the distributions of country object scores are not normal so that traditional confidence intervals theory is not applicable.

$\mathrm{CI}$ are obtained via a bootstrap procedure and results are reported in Figure 5.

Since NLPCA seems a good method to assess the overall level of satisfaction and it yields for each variable (aspect: "Equipment", "Sales Support", etc.) and each unit (respondent), the correlation between different aspects can be obtained in order to get "similar" aspects. The correlation matrix is reported in Table 5.

Table 5 shows that some aspects are more connected: for example, aspects concerning customer's support like Technical, Administrative Support, Training and Supplies \& Media seem more correlated than other aspects. 
Table 5. Correlation matrix among overall question quantifications.

\begin{tabular}{|l|c|c|c|c|c|c|c|}
\hline & Equipment & $\begin{array}{c}\text { Sales } \\
\text { Support }\end{array}$ & $\begin{array}{c}\text { Technical } \\
\text { Support }\end{array}$ & Training & $\begin{array}{c}\text { Supplies } \\
\& \text { Media }\end{array}$ & $\begin{array}{c}\text { Adm. } \\
\text { Support }\end{array}$ & $\begin{array}{c}\text { Terms, } \\
\text { Conditions } \\
\text { \& Pricing }\end{array}$ \\
\hline Equipment & 1.000 & 0.382 & 0.419 & 0.366 & 0.425 & 0.360 & 0.453 \\
\hline Sales Support & 0.382 & 1.000 & 0.385 & 0.243 & 0.270 & 0.321 & 0.470 \\
\hline Technical Support & 0.419 & 0.385 & 1.000 & 0.354 & 0.330 & 0.465 & 0.324 \\
\hline Training & 0.366 & 0.243 & 0.354 & 1.000 & 0.532 & 0.322 & 0.323 \\
\hline Supplies \& Media & 0.425 & 0.270 & 0.330 & 0.532 & 1.000 & 0.431 & 0.326 \\
\hline $\begin{array}{l}\text { Administrative } \\
\text { Support }\end{array}$ & 0.360 & 0.321 & 0.465 & 0.322 & 0.431 & 1.000 & 0.290 \\
\hline $\begin{array}{l}\text { Terms, Conditions } \\
\text { \& Pricing }\end{array}$ & 0.453 & 0.470 & 0.324 & 0.323 & 0.326 & 0.290 & 1.000 \\
\hline
\end{tabular}

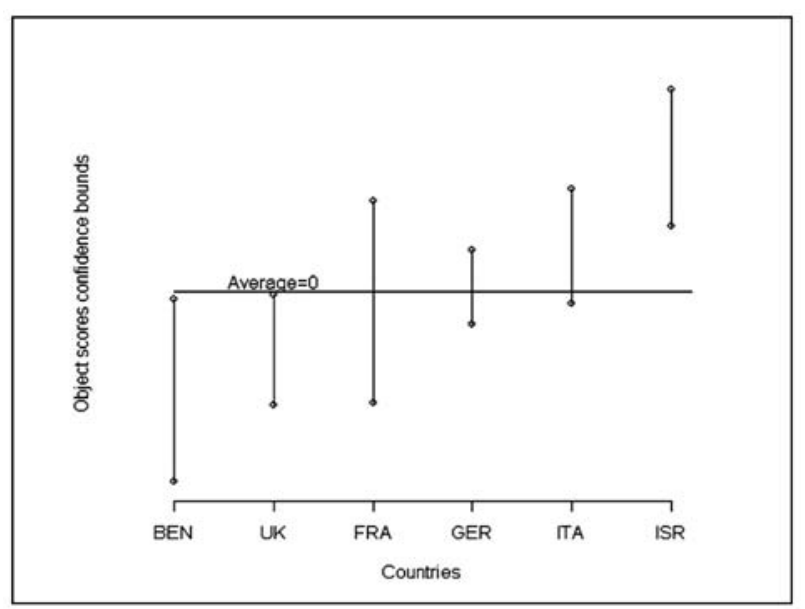

Figure 5. Bootstrap confidence intervals for the average scores

\section{Conclusions}

The aim of this paper is to show how the NLPCA method can fit the problem of the assessment of customer satisfaction. The analysis is based on the assumption that customer satisfaction constitutes a complex and latent dimension not directly discernible but identifiable through a plurality of directly observable variables whose categories (qualitative and ordinal) indicate the different levels and the different facets of satisfaction as perceived by customers.

The latent dimension is derived as a linear combination of the observed variables after a proper quantification of their ordinal categories. This technique enables to attain both optimal quantifications and optimal weights to be assigned to each variable in the construction of the linear combination which defines the latent dimension.

The procedure appears particularly fit for this goal as it allows the generation of a synthetic indicator of satisfaction which takes into account all the possible relationships among manifest variables. Furthermore, it is possible to carry out the analysis also in presence of missing data and to define the satisfaction level of a customer on the basis of her/his answers to the observed variables. Finally, by assigning a real number to each unit (customer), it makes possible to make analysis on the basis of these values, creating customers' profiles, forecasting new customers "types" and so on. Finally, if it is the case, it is possible to consider more than one latent dimension, enriching in this way the analysis. 


\section{References}

1. Andrich, D. (1978). A rating formulation for ordered response categories. Psycometrika, 43, 561-573.

2. Bertrand, M. and Mullainathan, S. (2001). Do people mean what they say? Implications for subjective survey data. Papers and proceedings of the hundred thirteenth annual meeting of the American economic association, economics and social behavior. The American Economic Review, 91(2), 67-72.

3. Bollen, K. A. (1989). Structural Equations with Latent Variables. Wiley, New York.

4. Cronbach, L. J. (1951). Coefficient alpha and the internal structure of tests. Psychometrika, 16, 297-334.

5. Efron, B. (1979). Bootstrap method: another look at the jackknife. Annals of Statistics, 7, $1-26$.

6. Efron, B. and Tibshirani, R. J. (1993). An Introduction to the Bootstrap. Chapman \& Hall/CRC, Boca Raton.

7. Ferrari, P. A. and Annoni, P. (2006). Missing Data in Optimal Scaling, Data Analysis, Classification and the Forward Search, eds. S. Zani, A. Cerioli, M. Riani and M. Vichi, Springer-Verlag, Berlin, 85-92

8. Ferrari P. A., Annoni P. and Salini S. (2005). A comparison between alternative models for environmental ordinal data: nonlinear PCA vs Rasch analysis. Proceedings of the $20^{\text {th }}$ International Workshop on Statistical Modelling, Sydney, 173-177.

9. Freeman, R. B. (1978). Job satisfaction as an economic variable. American Economic Association, 68(2), 135-141.

10. Gifi, A. (1990). Nonlinear Multivariate Analysis. Wiley, New York.

11. Heiser, W. J. and Meulman, J. J. (1994). Homogeneity analysis: exploring the distribution of variables and their nonlinear relationship, Correspondence Analysis in the Social Sciences: Recent Developments and Applications, eds. M. J. Greenacre and J. Blasius, Academic Press, Harcourt Brace \& Company New York, 179-209.

12. Jöreskog, K. G. (1970). A general method for analysis of covariance structure. Biometrika, 57, 2, 239-251.

13. Likert, R. (1932). A technique for the measurement of attitudes. Archives of Psychology, 140, 595-639.

14. Little, R. J. A. and Rubin, D. B. (2002). Statistical Analysis with Missing Data, $2^{\text {nd }}$ edition. Wiley Interscience, New York.

15. Masters, G. B. (1982). A Rasch model for partial credit scoring. Psychometrika, 47, 149-174

16. Michailidis, G. and De Leeuw, J. (1998). The Gifi system of descriptive multivariate analysis. Statistical Science, 13, 307-336.

17. Ramsey J. O. (1988). Monotone regression splines in Action. Statistical Science, 3, 425-441.

18. Rasch G. (1980). Probabilistic Models for Some Intelligence and Attainment Tests. University of Chicago Press, Chicago.

19. Tenenhaus, M., Esposito Vinzi, V., Chatelin, Y. M. and Lauro, C. (2005). PLS path modelling. Computational Statistics and Data Analysis, 48, 159-205.

20. Wold, H. (1982). Systems under indirect observation using PLS. A Second Generation of Multivariate Analysis: Methods, eds. C. Fornell, Praeger, New York, 325-347.

21. Wright, B. D. and Maesters, G. N. (1982). Rating Scale Analysis. MESA PRESS, Chicago.

Authors' Biographies:

Pier Alda Ferrari is Full Professor of Statistics at the Department of Economics, Business and Statistics at the Università degli Studi di Milano, Italy. Her main research interests are: multivariate statistical analysis, multilevel models, applications of statistics to sociology and economics.

Giancarlo Manzi is research fellow at the Department of Economics, Business and Statistics at the Università degli Studi di Milano, Italy. His main research interests are: sample theory, multilevel models, Monte Carlo methods, resampling methods, applications of statistics to linguistics, business statistics. 